\title{
Assemblage de fibres par tissage : analyse et simulation du comportement mécanique
}

\author{
Philippe Boisse $^{1, a}, B_{\text {assem Zouari }}^{2}$, François Dumont $^{2}$ et Alain Gasser ${ }^{2}$ \\ 1 Laboratoire de Mécanique des Contacts et des Solides, UMR CNRS 5514, INSA de Lyon, Bâtiment Jacquard, \\ rue Jean Capelle, 69621 Villeurbanne Cedex, France \\ 2 Laboratoire de Mécanique de Systèmes et des Procédés, UMR CNRS 8106, ENSAM, Université d'Orléans, \\ 8 rue Léonard de Vinci, 45072 Orléans Cedex, France
}

Reçu le 2 juillet 2004, accepté le 5 novembre 2004

\begin{abstract}
Résumé - Le comportement mécanique des renforts tissés utilisés dans les composites est fortement influencé par l'assemblage par tissage des mèches chaîne et trame. La rigidité principale du tissé est celle de tension. Le comportement en tension est non-linéaire compte tenu des liaisons entre les deux réseaux qui conduisent à des non-linéarités géométriques locales. Ce comportement en tension est analysé dans le présent travail par des essais de traction biaxiale et des simulations 3D de la maille élémentaire. La rigidité de cisaillement dans le plan des renforts tissés est faible jusqu'à un angle limite. On montre par des mesures optiques que ceci correspond à une phase de rotation de corps rigide de chaque mèche. Une deuxième phase correspondant à un blocage et un écrasement latéral des mèches conduit à des rigidités beaucoup plus fortes. On présente deux formes simplifiées de l'équation de la dynamique ne prenant en compte que les travaux virtuels de tension d'une part et de tension cisaillement d'autre part. On montre sur un exemple de simulation d'emboutissage d'un renfort tissé que la prise en compte du cisaillement est importante si l'on dépasse un angle limite.
\end{abstract}

Mots clés : Textiles / comportement multiéchelles / tension biaxiale / cisaillement plan / liaison par tissage / simulation de mise en forme

\begin{abstract}
Fibre link by weaving: analysis and simulation of the mechanical behaviour. The mechanical behaviour of woven reinforcements used in composites is mainly depending on weaving between warp and weft yarns. The principal stiffness of the fabric is the tensile rigidity. The tensile behaviour is non-linear accounting for the link between the yarns that leads to local geometrical non-linearities. This tensile behaviour is analysed in the present work by biaxial tensile tests and 3D finite element computations of the woven unit cell. The shear stiffness of fabrics is weak up to a limit angle. Optical measures show that this stage corresponds to a rigid body rotation of each yarn. A second stage in which the yarns are laterally crushed leads to much larger stiffnesses. Two simplified forms of the dynamic equation are presented. They only take into account tensile internal virtual work on one hand and tensile plus shear internal virtual works on the other hand. A fabric deep drawing simulation shows that it can be important to account for shear if a shear limit angle is exceeded.
\end{abstract}

Key words: Textiles / multiscale behaviour / biaxial tension / in plane shear / link by weaving / forming simulation

a Auteur correspondant : Philippe.Boisse@insa-lyon.fr 


\section{Nomenclature}

\begin{tabular}{|ll|}
\hline$\sigma=\sigma^{\alpha \beta} \mathbf{h}_{\alpha} \otimes \mathbf{h}_{\beta}$ & tenseur des contraintes de Cauchy \\
$\mathbf{h}_{1}, \mathbf{h}_{2}$ & vecteurs unitaires dans les directions chaîne et trame \\
$\mathbf{h}^{1}, \mathbf{h}^{2}$ & vecteurs contravariants associés $\left(\mathbf{h}_{\alpha} \cdot \mathbf{h}^{\beta}=\delta_{\alpha}^{\beta}\right)$ \\
$\boldsymbol{\eta}$ & déplacement virtuel avec $\boldsymbol{\eta}=0$ sur la frontière à déplacements imposés \\
$\mathbf{T}=T^{\alpha \beta} \mathbf{h}_{\alpha} \otimes \mathbf{h}_{\beta}$ & tenseur de tension \\
$\varepsilon=\varepsilon_{\alpha \beta} \mathbf{h}^{\alpha} \otimes \mathbf{h}^{\beta}$ & tenseur de déformation (gradient symétrique du déplacement) \\
${ }^{p} L_{1},{ }^{p} L_{2}$ & longueurs de la cellule élémentaire $p$ dans les directions trame et chaîne \\
$\rho$ & masse volumique \\
${ }^{\circ}$ & accélération \\
$A_{1}, A_{2}$ & surface de la section des mèches trame et chaîne \\
$T_{\text {ext }}(\boldsymbol{\eta})$ & travail virtuel des efforts extérieurs \\
$k=\varepsilon_{11} / \varepsilon_{22}$ & rapport de biaxialité \\
$E_{i}$ & module d'Young dans la direction $i$ \\
$E_{\varepsilon}$ & module transverse initial \\
$m, n$ & coefficients de la loi d'écrasement des mèches \\
${ }^{p} C$ & couple dû au cisaillement pour la cellule tissée $p$ \\
${ }^{p} \gamma(\boldsymbol{\eta})$ & rotation entre mèches chaîne et trame pour la cellule tissée $p$ \\
\hline
\end{tabular}

\section{Introduction}

De nombreux composites sont réalisés à partir de renforts tissés. Cet assemblage de fibres permet la fabrication de pièces à double courbure compte tenu de la cohésion apportée par le tissage. Par exemple les poutres à section en $\mathrm{Z}$ à simple courbure présentées figure 1a [1] sont réalisées par empilement de plis à renforts UD tandis que la pièce non-développable présentée figure 1b (et réalisée par Legrand à l'Ensam de Lille) nécessite l'emploi de renforts tissés pour les différents plis. Le comportement des renforts tissés (et de la plupart des tissus) est fortement affecté par la liaison entre les mèches chaîne et trame. Les ondulations des mèches et leurs variations lors de sollicitations membranaires conduisent à des nonlinéarités géométriques locales qui se traduisent en nonlinéarités de comportement global du matériau tissé. De même le contact entre les mèches d'un même réseau à partir d'un angle de distorsion augmente fortement la rigidité de cisaillement. La connaissance du comportement du tissé qui en résulte est nécessaire pour l'analyse de la réponse à des chargements de structures tissées en l'absence de résine ou lorsque cette résine n'est pas durcie car non-polymérisée ou rendue fluide par chauffage (thermoplastiques). En pratique, sont notamment concernées les structures tendues (génie civil, voile de bateaux...), mais aussi l'analyse des procédés de mise en forme tels que le RTM [2] où le formage du renfort se fait sans la résine, le drapage des préimprégnés où la résine n'est pas encore polymérisée [3], ou le thermoformage des composites à fibres continues et matrices thermoplastiques (CFRTP) [1]. Dans ce dernier cas, l'opération se fait à chaud de manière à rendre la matrice suffisamment fluide pour permettre les mouvements relatifs entre les renforts et obtenir une mise en forme.

Compte tenu de sa structure fibreuse, la principale rigidité d'un tissé est celle de tension et l'on présente dans ce travail une première formulation simplifiée du comportement mécanique du tissu ne prenant en compte que l'énergie de tension des mèches. Deux approches sont présentées pour analyser l'influence de l'assemblage des fibres par tissage sur le comportement mécanique en tension membranaire. Des essais de traction biaxiale mettent en évidence les non-linéarités à l'échelle macroscopique. Des simulations 3D de la maille élémentaire sont réalisées au niveau mésoscopique. Elles décrivent les liaisons entre chaîne et trame et le comportement fibreux de la mèche.

On introduit ensuite un terme de cisaillement plan dans le travail virtuel des efforts intérieurs. Si les déformations de cisaillement dans le plan correspondent à des efforts très faibles jusqu'à un angle limite, le blocage qui suit a des conséquences importantes sur la réponse mécanique du tissé, en particulier sur les plissements. Le comportement en cisaillement plan des renforts tissés est analysé par un essai de cadre articulé associé à une mesure des déformations par corrélation d'images. Cette dernière méthode permet des études à l'échelle microscopique (interne aux mèches) qui précisent les différentes étapes et expliquent la réponse macroscopique.

On montre sur une simulation de mise en forme les résultats obtenus à partir des deux formulations simplifiées mises en place.

\section{Comportement mécanique simplifié des renforts assemblés par tissage basé sur les surfaces de tension}

\subsection{Forme simplifiée du travail des efforts intérieurs}

Les fibres de verre, carbone et aramide présentent des caractéristiques mécaniques très intéressantes qui conduisent à chercher à les utiliser dans la fabrication de 
(a)

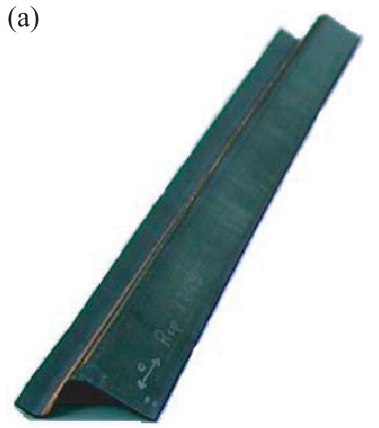

(b)

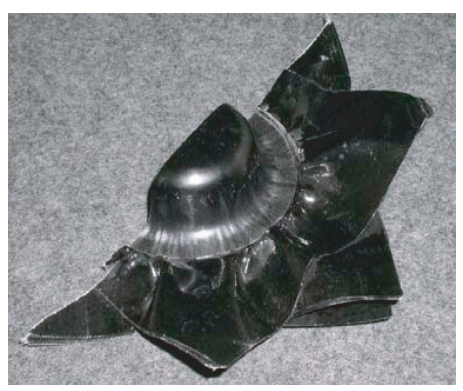

Fig. 1. (a) Poutre à section en $\mathrm{Z}$ (réalisation EADS), (b) pièce à double courbure.
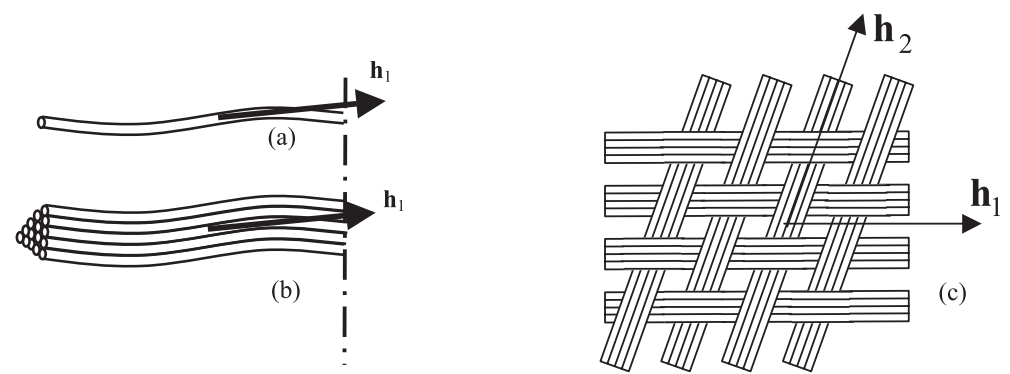

Fig. 2. (a) Fibre seule, (b) fil composé de fibres juxtaposées, (c) tissage de fils.

composants fortement sollicités dont on souhaite qu'ils soient légers. Le diamètre de ces fibres est très faible (5 à $7 \mu \mathrm{m}$ pour le carbone 5 à $25 \mu \mathrm{m}$ pour le verre) devant la longueur des fibres. En conséquences, ces fibres ne peuvent être sollicitées qu'en tension dans la direction de la fibre $\mathbf{h}_{1}$ (Fig. 2) :

$$
\boldsymbol{\sigma}=\sigma^{11} \mathbf{h}_{1} \otimes \mathbf{h}_{1} \quad \sigma^{11} \geq 0
$$

Les fibres sont assemblées pour former les fils (ou mèches). Ceux-ci peuvent être de structures différentes. Ceux qui sont utilisés pour les renforts des composites sont très souvent obtenus par juxtaposition d'un grand nombre de fibres (quelques milliers). Ceci permet un glissement relatif des fibres en cas de courbure du fil de sorte que les contraintes dans le fil (Fig. 2b) reste de la forme (1). Dans le cas de deux réseaux de fils dirigés suivant $\mathbf{h}_{1}$ et $\mathbf{h}_{2}$, l'état des contraintes peut être supposé de la forme :

$$
\boldsymbol{\sigma}=\sigma^{11} \mathbf{h}_{1} \otimes \mathbf{h}_{1}+\sigma^{22} \mathbf{h}_{2} \otimes \mathbf{h}_{2}
$$

La forme (2) suppose que l'on considère nulle l'influence du cisaillement dans le plan et en particulier les interactions entre les mèches voisines. On reviendra sur ces hypothèses à la section 4 . Pour un domaine composé de $n_{\text {cell }}$ cellules élémentaires tissées, une équation simplifiée de la dynamique est obtenue sous la forme $\forall \boldsymbol{\eta} / \boldsymbol{\eta}=0$ sur $\Gamma_{\mathrm{u}}$ :

$$
\begin{aligned}
\sum_{p=1}^{n_{\text {cell }}}{ }^{p} \varepsilon_{11}(\boldsymbol{\eta})^{p} T^{11 p} L_{1}+ & { }^{p} \varepsilon_{22}(\boldsymbol{\eta})^{p} T^{22}{ }^{p} L_{2} \\
& -T_{\text {ext }}(\boldsymbol{\eta})=\int_{\Omega} \rho \stackrel{\circ}{u} \boldsymbol{\eta} \mathrm{d} V
\end{aligned}
$$

où les tensions sont définies par :

$$
\begin{array}{r}
T^{11}=\int_{\mathrm{A}_{1}} \sigma^{11} \mathrm{~d} S, \quad T^{22}=\int_{\mathrm{A}_{2}} \sigma^{22} \mathrm{~d} S, \quad T^{11} \geq 0, \\
T^{22} \geq 0
\end{array}
$$

Bien que les procédés analysés soient pour la plupart quasistatiques, on s'intéresse à l'équation de la dynamique car, dans un souci d'efficacité numérique, les logiciels de simulation de la mise en forme utilisent actuellement des formulations en dynamique transitoire associées à un schéma explicite de type différences centrées.

\subsection{Surfaces de tension}

Les tensions $T^{11}$ et $T^{22}$ sont liées par le tissage aux deux déformations axiales dans les directions chaîne et trame

$$
T^{11}\left(\varepsilon_{11}, \varepsilon_{22}\right) \quad \text { et } \quad T^{22}\left(\varepsilon_{11}, \varepsilon_{22}\right)
$$

Ces deux fonctions qui définissent le comportement du tissé dans le cadre des hypothèses qui ont été faites sont les surfaces de tension du tissé. La surface est unique si le matériau est équilibré (même comportement sens chaîne et trame). L'objectif des chapitres suivants est la détermination de ces surfaces par deux approches différentes.

\section{Essais de traction biaxiale}

\subsection{Problème non-linéaire multi-échelles}

Des essais de traction pratiqués sur le tissu dans les directions des mèches (Fig. 6 pour un fil de carbone) 


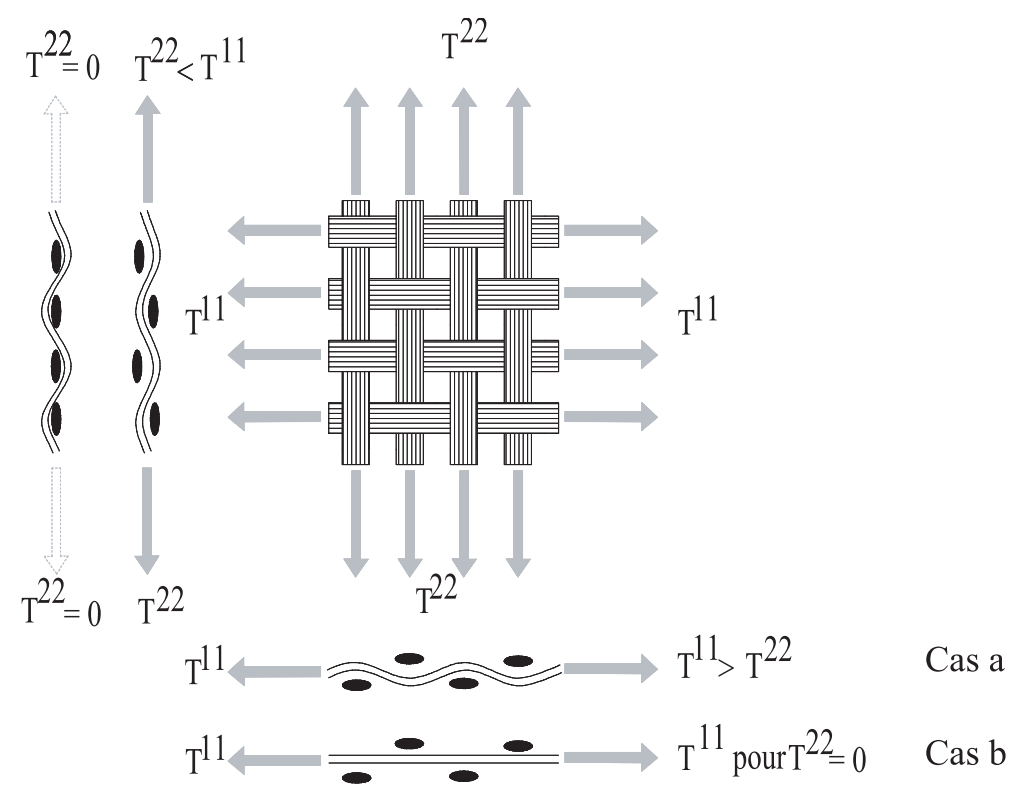

Fig. 3. Variations d'ondulation pour des tensions chaîne et trame.

montrent classiquement la présence d'une rigidification progressive suivie d'une linéarité du comportement. La non-linéarité de début de chargement s'explique par des phénomènes ayant lieu à des échelles inférieures. Du fait même du tissage et de l'alternance des fils, le tissu présente une ondulation naturelle de ses mèches. Or, sous l'effet de la tension, les fils ont tendance à devenir rectilignes. Dans un cas extrême où l'autre direction est laissée totalement libre de se déplacer, les mèches sollicitées deviennent totalement droites et les autres fortement ondulées (Fig. 3, cas b). Pour d'autres cas, intermédiaires, un état d'équilibre est atteint, où les deux directions subissent des variations d'ondulation (Fig. 3, cas a). Il apparaît clairement que ce phénomène est biaxial et que les deux réseaux sont en interaction. Constatés à l'échelle du motif élémentaire de tissage, ces phénomènes non-linéaires, d'origine géométrique, sont qualifiés de mésoscopiques. Leur effet est complété et amplifié par des phénomènes se manifestant au niveau des fibres (échelle microscopique). Sous l'effet des différents efforts de traction suivant l'axe des mèches, et de compression transverse au niveau du contact entre les deux réseaux, les filaments se réarrangent, et la forme de la section transverse des fils varie. Ces mouvements à l'intérieur d'un paquet de filaments sont à rapprocher des comportements du type câbles [4]. Les non-linéarités mises en jeu à cette échelle sont liées aux frottements entre les fibres et engendrent des non-linéarités de type géométrique à l'échelle mésoscopique. L'ensemble de ces phénomènes est à l'origine des non-linéarités matérielles observées à l'échelle macroscopique du tissu, lors de la déformation de celui-ci.

\subsection{Dispositif expérimental}

Afin d'appréhender le comportement spécifique des tissus et mettre en évidence les caractéristiques évoquées, des essais sont menés grâce à un dispositif capable de tester des matériaux tissés dans deux directions simultanément [5]. Le dispositif adopté repose sur le principe de deux losanges déformables [6] (Fig. 4). Des bielles de longueurs différentes permettent de faire varier les dimensions d'un des deux losanges et ainsi d'imposer divers rapports de déformations entre les directions chaîne et trame. Un système de réglage permet d'imposer un angle différent de $90^{\circ}$ entre les réseaux de mèches. Des capteurs de force placés directement derrière l'échantillon indiquent l'effort total dans chaque direction. Les mesures de déformations sont faites soit par méthode optique [7] soit par des extensomètres mécaniques. Une forme en croix est donnée à l'éprouvette (Fig. 4). Seule la partie centrale est tissée, et les extrémités sont maintenues par des talons en composite durcis qui permettent, par ailleurs, l'accrochage de l'échantillon dans le dispositif. Cette forme en croix est particulièrement bien adaptée aux tests biaxiaux des tissus qui présentent une très faible rigidité de cisaillement dans le plan (au moins lorsque les deux réseaux chaîne et trame sont perpendiculaires), contrairement aux matériaux plus continus tels que les métaux. On assure donc une bonne homogénéité du champ de déformation sur l'ensemble de la zone centrale tissée, y compris dans les coins qui posent problème dans le cas d'une rigidité de cisaillement importante dans le plan (cas des métaux).

\subsection{Sergé $2 \times 2$ de carbone}

Les fils utilisés sont identiques suivant la chaîne et la trame. Il s'agit de mèches composées d'environ 6000 fibres de carbone haute résistance assemblées sans torsion. Leur comportement en traction simple est quasi linéaire. Le tissu est quasi équilibré. On pourra donc ne présenter les résultats que suivant une direction. La figure 5 présente 


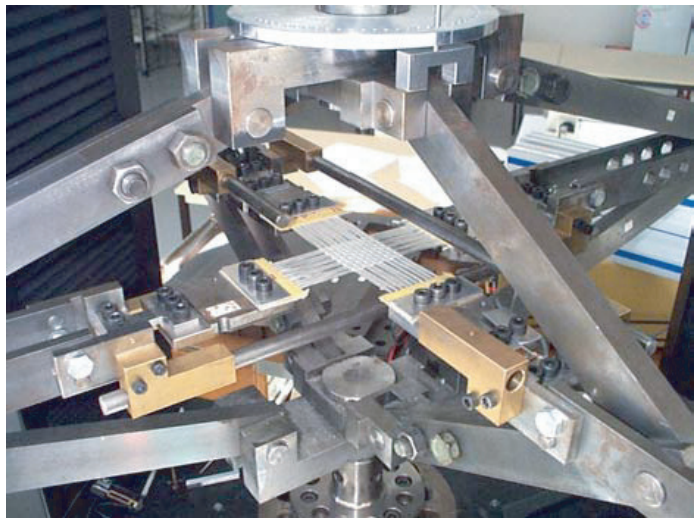

Fig. 4. Dispositif de traction biaxiale sur une éprouvette en croix.

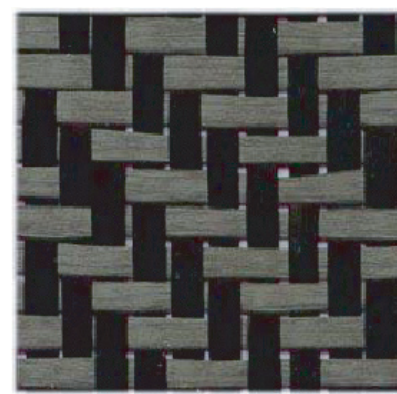

Densité (mèches $/ \mathrm{mm}): 0,35$

Embuvage (\%): 0,35

Ténacité d'un fil : $420 \mathrm{~N}$

Rigidité d'un fil : $54000 \mathrm{~N}$

Fig. 5. Sergé $2 \times 2$ de carbone.

les caractéristiques géométriques de ce tissu; la figure 6 montre les courbes de comportement. Bien que les mèches seules aient un comportement linéaire, les courbes de comportement tension-déformation du tissu sont très nettement non-linéaires aux faibles efforts puis linéaires aux efforts plus importants. Cette non-linéarité de comportement est une conséquence de phénomènes nonlinéaires se manifestant à des échelles inférieures (variations d'ondulation et écrasement des mèches). L'étendue de la zone non-linéaire est importante en regard de la déformation à rupture. Par ailleurs, elle dépend du rapport de déformations imposées, ce qui met ainsi en évidence le caractère biaxial du comportement des tissus, chaque direction ayant une influence sur le comportement de l'autre.

La zone de non-linéarité est maximale pour les essais libres dans la deuxième direction. En effet, dans la direction de traction les mèches tendent vers un état complètement rectiligne et ce, sous des efforts très faibles. Lorsque la rectitude du fil est atteinte, on retrouve le comportement des mèches seules. La valeur de la déformation correspondant à cette transition est significative de l'embuvage du tissu dans cette direction. Enfin, succède à la zone non-linéaire, une partie linéaire du comportement caractérisée par une rigidité proche de celle des mèches seules en traction simple.

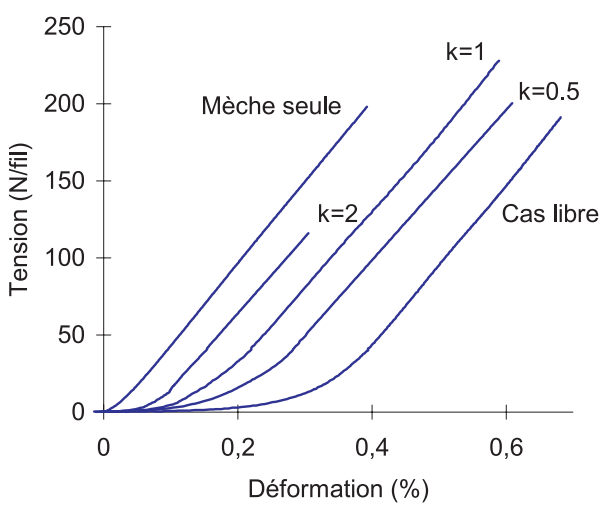

Fig. 6. Tension pour différents rapports $k=\varepsilon_{11} / \varepsilon_{22}$.

\subsection{Surfaces de tension}

Les courbes de tension pour différents rapports $k=$ $\varepsilon_{22} / \varepsilon_{11}$ permettent par extrapolation d'obtenir les surfaces de tension $T^{\alpha \alpha}\left(\varepsilon_{11}, \varepsilon_{22}\right)$ [8]. La surface obtenue pour le sergé de carbone $2 \times 2$ est présentée figure 7 . D'autres résultats expérimentaux sur différents renforts tissés équilibrés ou non peuvent être trouvés dans [5].

\section{Analyse éléments finis 3D de la maille tissée élémentaire}

Une autre approche pour la détermination des surfaces de tension consiste en la réalisation d'une simulation 3D d'une maille élémentaire de tissu [9]. Cette simulation peut en particulier permettre de :

- mieux comprendre le comportement du tissu à l'échelle de la mèche,

- obtenir le comportement d'un tissu sans avoir besoin de le fabriquer au préalable.

Pour ces simulations, la difficulté consiste à reproduire à l'aide d'éléments finis continus 3D le caractère fibreux des mèches (c'est-à-dire l'absence de certaines rigidités, en compression, flexion et cisaillement). Ce comportement spécifique est obtenu pour des modules de cisaillement quasi nuls, et des modules d'Young transverses faibles devant le module d'Young longitudinal (dans la direction des fibres). Il est également très important de bien suivre la fibre pendant son mouvement pour décrire le comportement. Deux approches ont été développées; l'une s'appuie sur l'utilisation de renforts (matériels) [9] et l'autre sur l'utilisation d'une dérivée objective basée sur la rotation de la mèche [10]. La quasi-absence de certaines rigidités (associée à de très faibles énergies) conduit à des instabilités numériques (modes parasites) qui sont éliminées grâce à l'ajout artificiel d'une rigidité en cisaillement, appelé « contrôle d'hourglass »[11]. Le comportement longitudinal de la mèche est obtenu par un essai de traction sur une mèche seule, tandis que le comportement transversal évolue en fonction des déformations transversale et longitudinale. Cette loi d'écrasement est supposée de la forme :

$$
E_{i}=E_{0}\left|\varepsilon_{i i}^{n}\right| \varepsilon_{11}^{m}+E_{\varepsilon}
$$



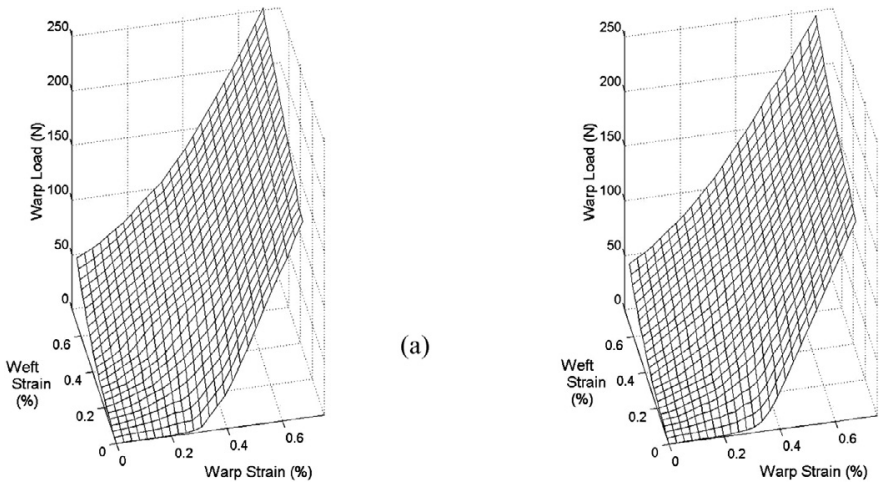

(b)

Fig. 7. Surface de tension $T\left(\varepsilon_{11}, \varepsilon_{22}\right)$ pour le sergé $2 \times 2$ de carbone. (a) essais, (b) analyse EF 3D.

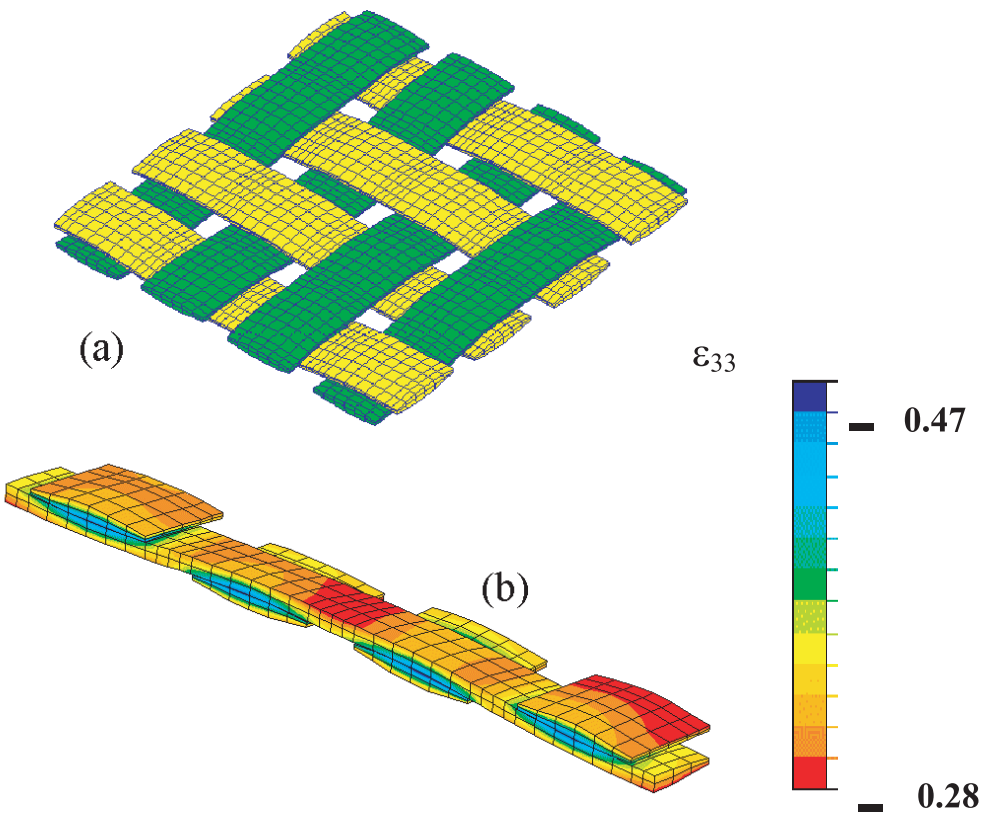

Fig. 8. Simulation 3D d'un essai de traction équibiaxiale sur une maille élémentaire de sergé $2 \times 2$ de verre : (a) maillage, (b) déformation transverse.

où $E_{0}, m$ et $n$ sont trois paramètres du matériau, l'indice 1 représente la direction de la mèche et $i$ une direction perpendiculaire ( $i$ valant 2 ou 3$)$. $E_{\varepsilon}$ est le module transverse initial (ayant une valeur très faible). Du fait de la grande difficulté à réaliser un essai de compression transverse sous tension variable, c'est un essai de traction équibiaxiale $(k=1)$ qui a permis d'identifier les trois paramètres de la loi d'écrasement, en utilisant une méthode inverse basée sur l'algorithme de LevenbergMarquardt [12]. Les mèches de chaîne et de trame étant en contact, ces phénomènes de contact et de frottement sont pris en compte à l'aide d'une approche de type maîtreesclave. Enfin, c'est la maille élémentaire minimale qui est étudiée : les conditions aux limites représentent les conditions de périodicité, ainsi que les éventuelles symétries. On présente figure 8 l'analyse d'une maille élémentaire du sergé de carbone introduit figure 5 sous une traction biaxiale à $k=1$. La surface de comportement obtenue par la simulation est en bonne cohérence avec celle donnée par les essais biaxiaux (Fig. 7). La figure 8 montre que l'écrasement des mèches est important (45 \% maxi). C'est cet écrasement qui conduit aux non-linéarités de comportement pour les tractions à $k=1$. C'est un phénomène important du comportement dans le plan d'un renfort tissé et il est nécessaire de le décrire de la façon la plus judicieuse possible dans l'analyse au niveau de la maille. D'autres résultats de simulations 3D de mailles élémentaires sont réalisées dans $[9,13]$. Une étude de même nature est réalisée sur une maille de renfort tricoté dans [10].

\section{4 Étude du comportement en cisaillement dans le plan}

\subsection{Distorsion d'un tissu dans son plan}

Les plus fortes rigidités d'un renfort tissé sont celles de tension. Ce sont elles qui guident principalement les changements de forme par exemple lors d'une fabrication d'un 


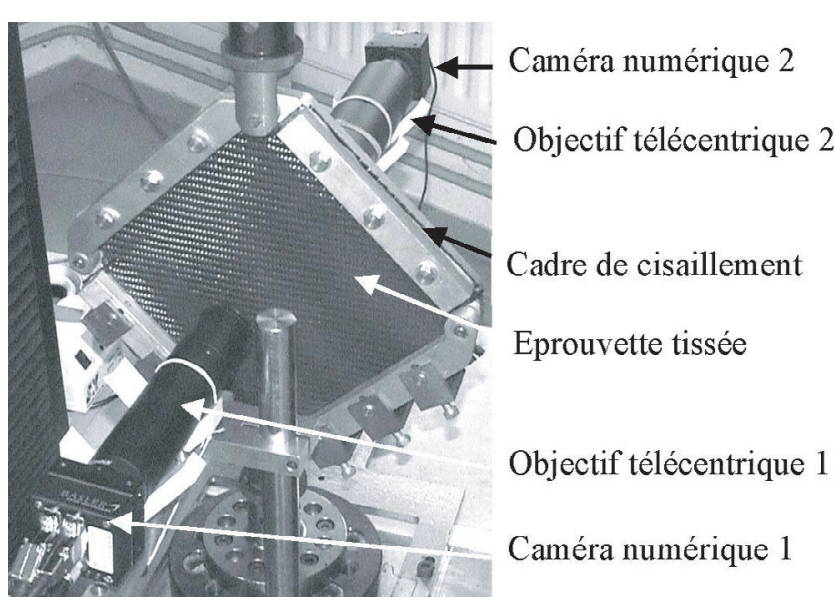

Fig. 9. Cadre de cisaillement équipé d'un système de mesure optique des déformations.

composite par formage du renfort. La rigidité en cisaillement plan est faible (au moins dans un premier temps) et il résulte de grandes distorsions qui sont le mode membranaire principal de formage. Si la rigidité en cisaillement plan est faible on peut s'intéresser à ce comportement mécanique comme complément au comportement en tension. Par ailleurs, la rigidité en cisaillement peut être augmentée si le tissu est enduit d'une matrice (thermodurcissable non-polymérisée ou thermoplastique au-dessus de la température du fusion) voire très augmentée si l'on dépasse l'angle limite ou angle de blocage en cisaillement. Les études concernant le comportement en cisaillement plan sont nombreuses, probablement car il s'agit du mode de déformation majeur des tissus [14-17]. Dans ce chapitre, on analyse expérimentalement le comportement en cisaillement plan de renforts tissés secs en se plaçant à la fois à l'échelle macroscopique et à l'échelle interne aux mèches (microscopique). Un terme de cisaillement est ensuite introduit dans l'équation de la dynamique.

\subsection{Analyse expérimentale par cadre de cisaillement}

Le cadre de cisaillement (présenté Fig. 9) est basé sur le principe d'un cadre déformable à quatre cotés égaux et articulés dont les directions sont celles des mèches $\mathrm{du}$ tissu. Différentes études tendent à montrer que ce dispositif est le plus satisfaisant pour imposer un état de cisaillement homogène dans un renfort tissé [18]. Le cadre est monté sur une machine de traction conventionnelle. Une pré-tension est appliquée sur les mèches avant le cisaillement. La surface active des échantillons est $200 \times 200 \mathrm{~mm}^{2}$.

\subsection{Mesures optiques des déformations}

Un système optique à deux échelles (Fig. 9) acquière des images de l'ensemble du cadre (échelle macro) ou seulement d'un faible nombre de mailles $(12,4 \times$ $\left.9,5 \mathrm{~mm}^{2}\right)$. Le domaine de mesure peut être restreint à

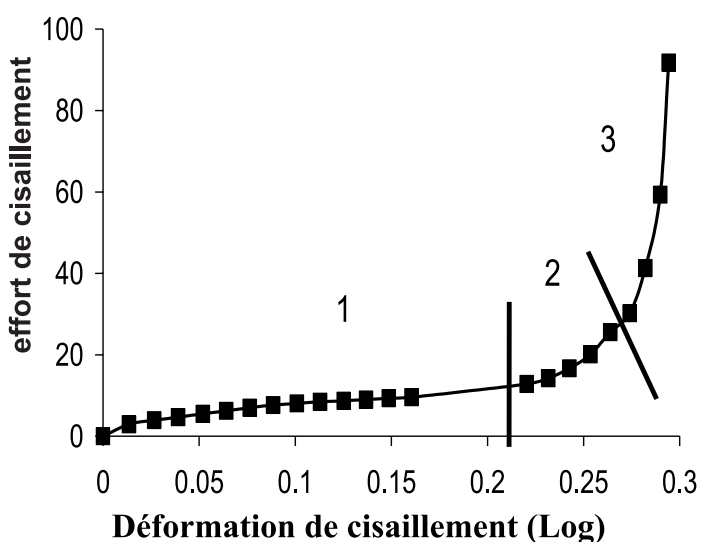

Fig. 10. Courbe de comportement en cisaillement plan d'un taffetas de verre.

une mèche unique (échelle micro). Deux images (macro et micro) sont prises au même temps en utilisant des caméras de part et d'autre de l'échantillon. Ces images sont utilisées pour calculer les champs de déplacements et de déformations grâce à une méthode de corrélation d'images [19]. Les mesures macroscopiques donnent la mesure du cisaillement en fonction de la force sur le cadre. Comme il s'agit de mesures de champs, elles permettent de vérifier l'homogénéité du cisaillement dans l'éprouvette. Ceci a conduit à adopter des systèmes de fixation de l'éprouvette sur les bras du cadre satisfaisant de ce point de vue $[20,21]$.

\subsection{Analyses micro-macro}

La figure 10 présente la mesure de l'effort de cisaillement en fonction de la distorsion. L'éprouvette est réalisée en taffetas (motif de tissage le plus simple) de verre dont les caractéristiques sont les suivantes : densité des mèches $n=0,25$, embuvage : $S=0,5 \%$, densité surfacique : $W=600$ g.m ${ }^{-2}$, densité linéaire : $w=1200$ tex ( 1 tex $=1$ g. $\left.\mathrm{km}^{-1}\right)$. La courbe de réponse en cisaillement peut être divisée en trois zones. La figure 11 montre pendant la phase 1, les déplacements à l'échelle micro, c'est-à-dire à l'intérieur de la mèche. On retranche pour cela le déplacement moyen à tous les déplacements mesurés. On constate que les mèches sont soumises à une rotation de corps rigides qui globalement, d'un point de vue macroscopique, se traduit par un cisaillement du tissu (vu comme milieu continu). Il n'y a pas de cisaillement à l'intérieur des mèches. Ceci explique que le cisaillement du tissu corresponde à des efforts faibles puisque seuls les frottements de rotation entre réseaux chaîne et trame s'opposent à la distorsion. Le passage à la zone 2 correspond à l'angle limite ou angle de blocage en cisaillement. À partir de cet angle les mèches arrivent en contact avec leurs voisines et sont comprimées latéralement (Fig. 12), d'abord partiellement (zone 2) puis complètement (zone 3) ce qui explique l'augmentation très rapide de l'effort de cisaillement et donc de la rigidité en cisaillement. En pratique, dans la zone 3, des 


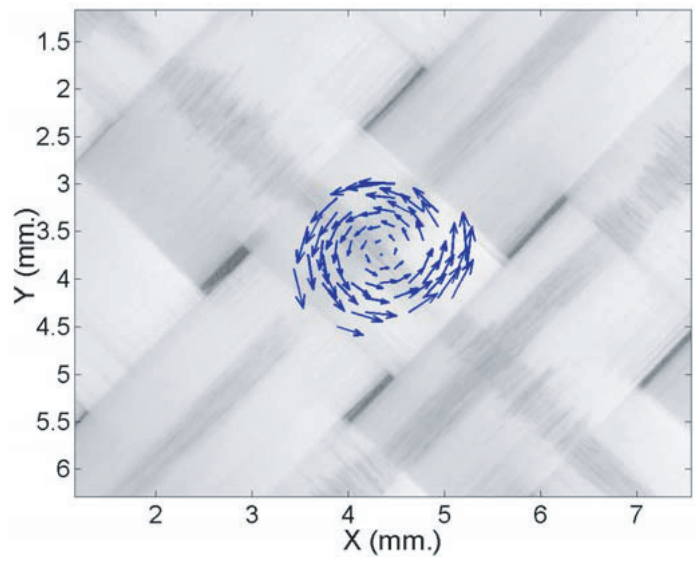

Fig. 11. Déplacements dans une mèche de trame pendant la phase 1 .

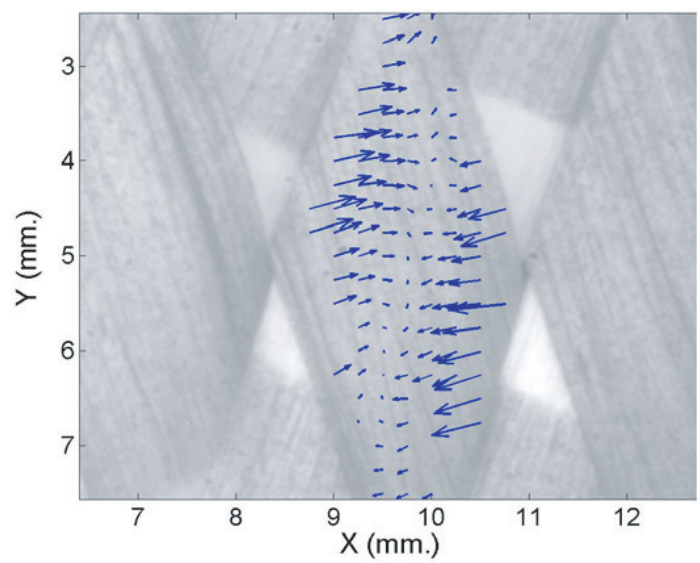

Fig. 12. Déplacements à l'échelle micro après l'angle de blocage.

plissements apparaissent, provoqués par le blocage en cisaillement.

\subsection{Prise en compte du cisaillement dans l'équation de la dynamique}

L'équation de la dynamique simplifiée (3) ne prend en compte que le travail virtuel des efforts intérieurs de tension dans chaque cellule tissée.

La forme (6) prend en compte le cisaillement dans le plan

$$
\begin{aligned}
& \sum_{p=1}^{n_{\text {cell }}}{ }^{p} \varepsilon_{11}(\boldsymbol{\eta}){ }^{p} T^{11}{ }^{p} L_{1}+{ }^{p} \varepsilon_{22}(\boldsymbol{\eta})^{p} T^{22}{ }^{p} L_{2} \\
& +\sum_{p=1}^{n_{\text {cell }}}{ }^{p} \gamma(\boldsymbol{\eta})^{p} C-T_{\text {ext }}(\boldsymbol{\eta})=\int_{\Omega} \rho \stackrel{\circ}{u} \boldsymbol{\eta} \mathrm{d} V
\end{aligned}
$$

${ }^{p} C$ est le couple dû au cisaillement pour la cellule tissée $p$ et ${ }^{p} \gamma(\boldsymbol{\eta})$ la rotation entre mèches chaînes et trames pour le champ de déplacement virtuel $\boldsymbol{\eta}$. La courbe effortdistorsion telle que présentée figure 10 permet de relier le

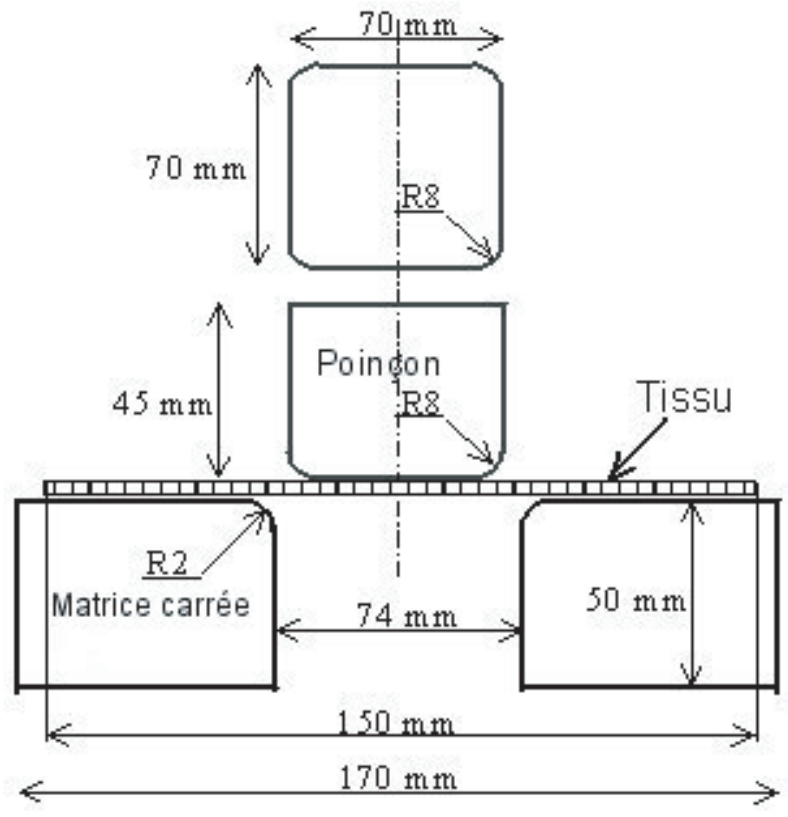

Fig. 13. Emboutissage d'une boîte carrée : géométrie des outils.

couple à la rotation chaîne-trame [22]. Les formes simplifiées de l'équation de la dynamique prenant en compte le travail intérieur virtuel de tension uniquement (3) et de tension et cisaillement (7) permettent l'une et l'autre de construire des éléments finis simplifiés spécifiques aux matériaux tissés et qui peuvent être utilisés dans un contexte de dynamique explicite $[13,22]$. On montre dans l'exemple suivant les résultats obtenus avec et sans cisaillement dans un exemple d'emboutissage.

\subsection{Emboutissage d'une boîte carrée}

Ce test présenté figure 13 est un benchmark classique pour l'emboutissage. Il a été proposé comme test à la conférence Numisheet 93 [23]. Compte tenu du caractère fortement non-développable de la forme, ce test est sévère, en particulier pour le formage des tissés, car il demande de grandes variations d'angle entre chaîne et trame dans les rayons des congés de la boîte carrée. Si l'on diminue ces rayons on obtient rapidement des distorsions chaîne-trame nécessaires à la mise en forme supérieures à l'angle limite du tissu utilisé. La mise en forme d'un taffetas (correspondant aux caractéristiques données en 4.4) est réalisée en utilisant l'approche en tension uniquement (Éq. (3)) et l'approche en tension et cisaillement (Éq. (7)). On présente figure 14 dans les deux cas la déformée du renfort en fin de mise en forme. Compte tenu du caractère très non-développable de la surface formée, l'angle limite de cisaillement du taffetas utilisé est largement dépassé. Ce qui conduit à des résultats assez différents pour les deux approches. Il n'y a pas de plis avec l'approche en tension car rien n'est source d'instabilité dans celle-ci. Par contre des plis se développent dans la seconde approche, créés par le blocage en cisaillement qui conduit 

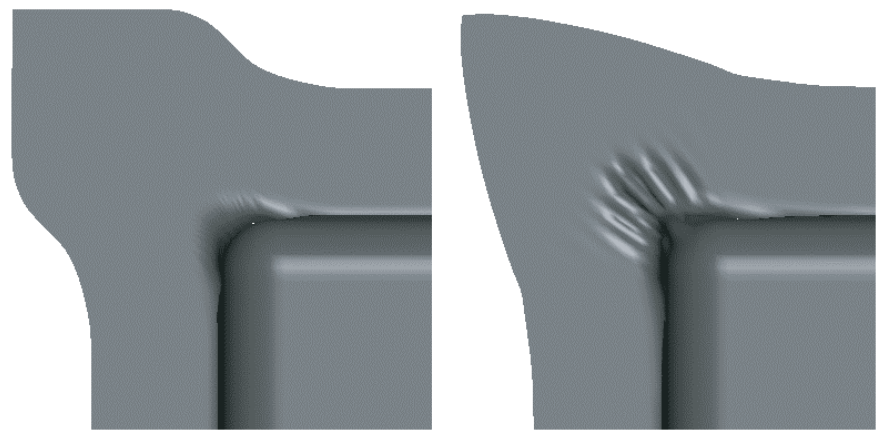

Fig. 14. Déformée du renfort tissé en fin de descente du poinçon. Formulation en tension (à gauche); Tension + cisaillement (à droite).

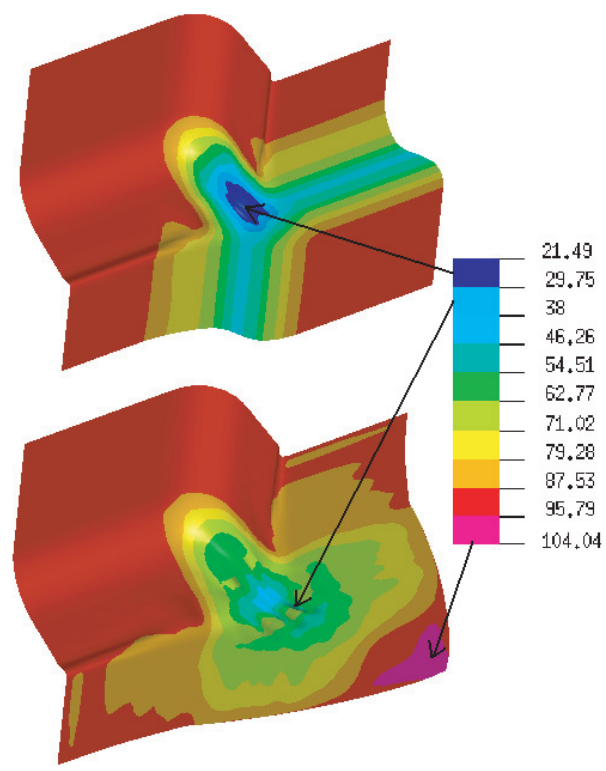

Fig. 15. Angles chaîne-trame. Formulation en tension (en haut); Tension + cisaillement (en bas).

à des solutions hors plan pour réduire ce cisaillement. On constate bien figure 15 que les angles sont nettement réduits dans le cas de la prise en compte du cisaillement. Cette deuxième approche est dans ce cas là plus conforme à la réalité. Ceci est lié au fait que l'on simule un cas où le formage demande de dépasser l'angle limite de cisaillement. Ce qui ne doit pas être le cas pour obtenir une pièce réussie. On peut également noter que l'approche en tension seule détecte également le dépassement de l'angle limite. L'apport du terme de cisaillement se situe principalement dans la description de la déformée après l'apparition des plissements.

\section{Conclusion}

Que ce soit en tension ou en cisaillement dans le plan, le comportement mécanique d'un tissé est fortement conditionné par l'assemblage des fibres par tissage. En tension, le comportement est fonction du changement de forme des ondulations créées par le tissage.
Les non-linéarités ainsi obtenues sont fonction du rapport de biaxialité. Les surfaces qui quantifient ce comportement en tension ont été obtenues par des essais de traction biaxiale et des calculs éléments finis $3 \mathrm{D}$ sur la maille élémentaire. Les non-linéarités de contact au niveau local influent également fortement sur le comportement en cisaillement du tissu. La rigidité est faible pour les angles modérés. Il a été montré par des mesures optiques que les mèches sont en rotation de corps solide. Lorsque les mèches entrent en contact avec les mèches voisines, elles sont comprimées et la rigidité de cisaillement augmente fortement. Des simulations de formage peuvent être réalisées à partir de formulations simplifiées basées sur les deux modes de déformations précédents. Si la transformation est avant tout définie par les tensions, la prise en compte du cisaillement est nécessaire quand on dépasse l'angle limite de cisaillement. Il reste à bien identifier les cas où seule la prise en compte de la tension est suffisante et ceux pour lesquels la prise en compte du cisaillement dans le plan est nécessaire. Dans ce dernier cas, la non-prise en compte de la flexion (comme cela a été fait ici) est une hypothèse qui mériterait d'être validée, en particulier vis-à-vis de la forme des plissements obtenus.

\section{Références}

[1] A. Cheruet, D. Soulat, P. Boisse, E. Soccard, S. Maison-le Poec, Analysis of the interply porosities in thermoplastic composites forming processes, Int. J. Forming Processes 5 (2002) 247-258

[2] D. Carronnier, D. Gay, Approche intégrée du RTM, Revue des Composites et des Matériaux Avancés 6 (1996)

[3] S. Bickerton, P. Simacek, S.E. Guglielmi, S.G. Advani, Investigation of draping and its effect on the mold filling process during manufacturing of a compound curved composite part, Composites Part A 28 (1997) 801-816

[4] D. Durville, Modélisation du comportement mécanique de câbles métalliques, Actes du $3^{\text {e }}$ Colloque en Calcul des Structures, Giens, France, 1997, pp. 139-144

[5] K. Buet-Gautier, P. Boisse, Experimental analysis and modeling of biaxial mechanical behavior of woven composite reinforcements, Experimental Mechanics 41 (2001) 260-269

[6] G. Ferron, Dispositif de traction biaxiale DAX2, Document Techmetal, Mézières-Lès-Metz, 1992

[7] J. Launay, F. Lahmar, P. Boisse, P. Vacher, Strain measurement in tests on fibre fabric by image correlation method, Advanced Composite Letters 11 (2002) 7-12

[8] G. Hivet, Modélisation mésoscopique pour le comportement bi-axial et la mise en forme des renforts de composites tissés, Thèse, Université d'Orléans, 2002

[9] A. Gasser, P. Boisse, S. Hanklar, Mechanical Behaviour of Dry Fabric Reinforcements, 3D simulations versus Biaxial Tests, Computational Materials Science 17 (2000) 7-20

[10] B. Hagège, Simulation du comportement mécanique des milieux fibreux en grandes transformations : application aux renforts tricotés, Thèse, ENSAM Paris, 2004

[11] D.P. Flanagan, T. Belytschko, A uniform strain hexahedron and quadrilateral with orthogonal hourglass control, Int. J. Numerical Methods in Engineering 17 (1981) 679-706 
[12] D.S. Schnur, N. Zabaras, An inverse method for determining elastic material properties and a material interface, Int. J. Numerical Methods in Engineering 33 (1992) 2039-2057

[13] P. Boisse, A. Gasser, G. Hivet, Analyses of fabric behaviour : determination of the biaxial tension-strain surfaces and their use in forming simulations, Composites Part A 32 (2001) 1395-1414

[14] T.M. McBride, J. Chen, Unit-cell geometry in plainweave fabrics during shear deformations, Composites Science and Technology 57 (1997) 345-351

[15] A.G. Prodromou, J. Chen, On the relationship between shear angle and wrinkling of textile composite preforms, Composites Part A 28 (1997) 491-503

[16] G.B. McGuinness, C.M.O. Bradaigh, Development of rheological models for forming flows and picture-frame shear testing of fabric reinforced thermoplastic sheets, J. Non-Newtonian Fluid Mechanics 73 (1997) 1-28

[17] G.B. McGuinness, C.M.O. O'Bradaigh, Characterisation of thermoplastic composite melts in rhombus-shear : the picture-frame experiment, Composites Part A 29 (1998) $115-132$
[18] A.C. Long, Characterisation and modelling of fabric deformation during forming of textile composites, Int. J. Forming Processes 4 (2002) 285-301

[19] P. Vacher, S. Dumoulin, R. Arrieux, Determination of the forming limit diagram from local measurement using digital image analysis, Int. J. Forming Processes 2 (1999) 395-408

[20] F. Dumont, Expérimentations et modèles de comportement de renforts composites tissés, Thèse, Université Paris 6, 2003

[21] D.S. Lussier, J. Chen, Material characterization of woven fabrics for thermoforming of composites, J. Thermoplastic Composite Materials 15 (2002) 497-509

[22] B. Zouari, F. Dumont, J.L. Daniel, P. Boisse, Analyses of woven fabric shearing by optical method and implementation in a finite element program, Proceedings of 6th Esaform, Salerno, Italy, 2003, pp. 875-878

[23] Numerical simulation of 3D sheet metal forming processes - Verification of simulation with experiments, Proceedings of NUMISHEET '93, Tokyo, Japan, 1993 